\title{
The fate of optical excitations in small polyhedral ZnS clusters: A theoretical study of the excitation and localization of electrons in $Z_{4} S_{4}$ and $Z n_{6} S_{6}$
}

\author{
Martijn A. Zwijnenburg, ${ }^{1, a)}$ Carmen Sousa, ${ }^{1}$ Francesc Illas, ${ }^{1}$ and Stefan T. Bromley ${ }^{1,2}$ \\ ${ }^{1}$ Departament de Química Física and Institut de Química Teòrica i Computacional (IQTCUB), Universitat de \\ Barcelona, E-08028 Barcelona, Spain \\ ${ }^{2}$ Institució Catalana de Recerca i Estudis Avançats (ICREA), 08010 Barcelona, Spain
}

(Received 3 September 2010; accepted 20 December 2010; published online 11 February 2011)

\begin{abstract}
We explore the excited state energy landscape of small polyhedral zinc sulfide clusters $\left(\mathrm{Zn}_{4} \mathrm{~S}_{4}\right.$ and $\mathrm{Zn}_{6} \mathrm{~S}_{6}$ ) using time-dependent density functional theory and correlated wave function based methods. We predict the optical absorption and photoluminescence spectra of the polyhedral clusters and demonstrate that, upon relaxation of the excited state, these nanostructures break symmetry and an electron and a hole localize on a small number of $\mathrm{Zn}$ (electron) and $\mathrm{S}$ (hole) centers. We further test several exchange-correlation potentials for their ability to recover the correlated wave function description of the excited state. Finally, we discuss how the degeneracy of excited states in nanostructures, such as those considered here, results in a Jahn-Teller distortion of the excited state geometry, and how numerical problems arising from this can be circumvented by starting the optimization of excited states from structures distorted along the ground state vibrational normal modes. (C) 2011 American Institute of Physics. [doi:10.1063/1.3536501]
\end{abstract}

\section{INTRODUCTION}

Nanostructures of metal chalcogenides (e.g., ZnS, ZnSe, and CdS) are experimentally known to interact strongly with light. More specifically, they are known to absorb and re-emit light in the ultraviolet or visible range of the electromagnetic spectrum. The latter process, referred to as photoluminescence (PL), in which light of a lower wavelength than the absorbed light is re-emitted, underlies the application of metal chalcogenide nanostructures in (bio)medical imaging. ${ }^{1-3}$ Other light related uses of metal chalcogenide nanostructures include photovoltaics, ${ }^{4-7}$ nanolasers, ${ }^{8,9}$ and photocatalysts. ${ }^{10-14}$ Despite the clear importance of these applications, theoretical knowledge of the photochemical and photophysical processes that underlie them is still rather limited. Some previous computational studies have focused on the optical absorption spectra of nanostructures (see below), but to our knowledge, no detailed theoretical understanding of what happens upon relaxation of the excited state (e.g., localization of the excited state, PL) exists. This lack of theoretical work is not surprising as efficient computational methods to probe the relaxation of excited states in nanosized materials, such as time-dependent density functional theory (TD-DFT) with analytical forces, ${ }^{15-17}$ have been only available for less than a decade. Furthermore, most applications have focused on organic molecules, ${ }^{17}$ although there has been some work ${ }^{18-22}$ on inorganic nanostructures. Herein we start addressing this knowledge gap and study the excited state behavior of $\mathrm{ZnS}$ clusters that are small enough so that

\footnotetext{
a) Author to whom correspondence should be addressed. Electronic mail: m.zwijnenburg@ucl.ac.uk. Present address: Christopher Ingold Laboratories, Department of Chemistry, University College London, 20 Gordon Street, London WC1H 0AJ, United Kingdom.
}

they can not only be studied by TD-DFT but also by correlated wave function based methods such as the accurate complete active space second-order perturbation theory (CASPT2)..$^{23,24}$ This approach allows for a proof of principal study whereby correlated wave function methods and TD-DFT can be compared, allowing us to benchmark the latter, more computationally efficient approach, for future work on larger nanostructures.

$\mathrm{ZnS}$ nanostructures of different shapes and aspect ratios have been prepared experimentally using a variety of methods, ranging from laser ablation under high vacuum to wet emulsion chemistry. Optical absorption spectra of $\mathrm{ZnS}$ nanostructures have been reported by several authors ${ }^{25-31}$ who all report absorption features in the $250-350 \mathrm{~nm}(5.0-3.5 \mathrm{eV})$ range, i.e., in the ultraviolet and at shorter wavelengths. Chen and co-workers also report an additional absorption feature at longer wavelengths in the visible range, with a maximum depending on the particle size between 450 and $550 \mathrm{~nm}(2.8-$ $2.3 \mathrm{eV}$ ), that they identify with surface states. ${ }^{32}$ Dhas et al. report an even longer wavelength broad absorption feature with a maximum around $600 \mathrm{~nm}(2.1 \mathrm{eV}) .{ }^{33}$ It is unclear if the latter absorption features are absent for the samples prepared by the other authors as a result of different preparation and/or measurement conditions, or simply lay outside of the spectral range studied. The PL spectrum of $\mathrm{ZnS}$ nanostructures has also been measured by several groups and generally consists of a broad peak that, depending on the preparation and measurement conditions, lies typically between 420 and $520 \mathrm{~nm}$ (3.0-2.4 eV)..$^{27,30-35}$ Theoretically, the geometries of the most stable $(\mathrm{ZnS})_{\mathrm{n}}$ nanostructures have been predicted by several authors $^{36-42}$ and for $6 \leq n \leq 50$ are reported to be polyhedral and cagelike, with rare exceptions (e.g., $n=13$ ). For $n$ $<6$ the predicted geometries of the lowest energy structures 


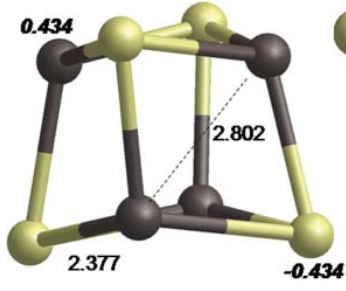

A

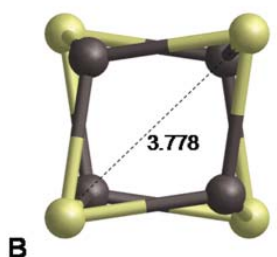

B

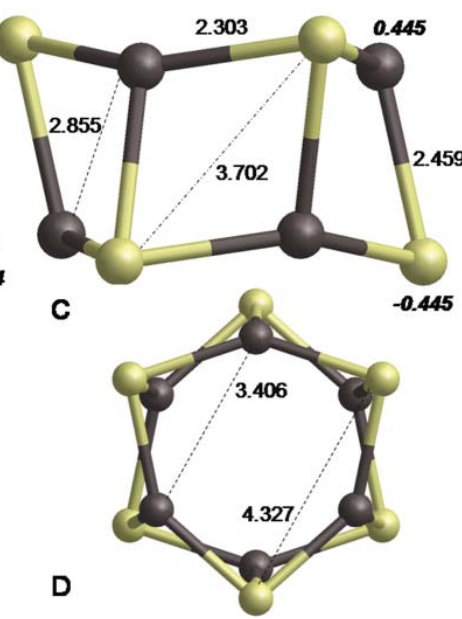

FIG. 1. Views of the B3LYP/def2-TZVP optimized $\mathrm{Zn}_{4} \mathrm{~S}_{4}$ [side (a) and top (b)] and $\mathrm{Zn}_{6} \mathrm{~S}_{6}$ [side (c) and top (d)] polyhedral nanoclusters studied here, including characteristic distances and Mulliken charges (in bold).

are rings but for $\mathrm{n}=4$ a polyhedral cluster (a cube) lies low in energy above the flat ring minimum energy geometry. Matxain and co-workers used TD-DFT and the multireference configuration interaction (MRCI) method ${ }^{43}$ to calculate the optical absorption spectra of both small $(\mathrm{n}=1-3$, TD-DFT and MRCI) (Refs. 44 and 45) and larger clusters ( $\mathrm{n}=4-47$, TD-DFT), ${ }^{45,46}$ with large-core pseudopotentials on $\mathrm{Zn}$ and $\mathrm{S}$ centers, and minimal basis-set for the larger structures (lacking $p$ and $d$ functions).

In the following study we present the results of our explorations of the excited state energy landscape of the $\mathrm{Zn}_{4} \mathrm{~S}_{4}$ cube and $\mathrm{Zn}_{6} \mathrm{~S}_{6}$ hexagonal prism polyhedral clusters (see Fig. 1). More specifically, we demonstrate that upon relaxation of the excited state these nanostructures break symmetry and an excited electron and hole localize on a small number of $\mathrm{Zn}$ (electron) and $\mathrm{S}$ (hole) centers. We further show that TD-DFT with the widely used B3LYP exchange-correlation (XC) potential gives results that are comparable to those obtained with correlated wave function based methods and that the contributions to the Stokes shift of both the energetic stabilization of the excited state and the energetic destabilization of the ground state are large and significant. Finally, we discuss the problem of degeneracy in excited states and our method of dealing with this.

\section{COMPUTATIONAL DETAILS}

The excited state properties of the $\mathrm{ZnS}$ clusters were calculated using a five-step approach. First, the ground state geometries of the clusters were optimized using ground state density functional theory (DFT), typically using the B3LYP XC potential ${ }^{47}$ (see below). Second, the harmonic frequencies at the optimized ground state geometries were calculated using the same DFT method to verify that the optimized structures correspond to proper minima on the ground state energy surface. Third, the excitations at the optimized ground state geometry were calculated using TD-DFT. Fourth, the lowest singlet excited state of each cluster was relaxed using TD-DFT to obtain its minimum energy geometry. Finally, frequency calculations on the excited state minima were performed to verify that they correspond to proper minima on the excited state energy surface.

All DFT and TD-DFT calculations were performed using the TURBOMOLE 5.10 code, which includes analytical forces for both ground and excited state calculations, and, unless otherwise specified, employed the hybrid B3LYP XC potential. For some specific cases and where explicitly stated in the text, further calculations were also performed using the $\mathrm{PBE},{ }^{48}$ generalized gradient approximation (GGA), and the BHLYP (Ref. 49), hybrid, XC potentials to probe the effect of the amount of Fock exchange on the results obtained. In all DFT/TD-DFT calculations the electron density was expanded in one of three different all-electron triple-zeta quality or higher basis-sets: aug-ccpVTZ (Refs. 50 and 51), def2-TZVP (Ref. 52), and def2QZVP (Ref. 52). Finally, all geometries, both those of the ground and excited states, were relaxed until the maximum norm of the Cartesian gradients was smaller than $1 \times 10^{-6}$ Hartree/Bohr.

Further calculations on the excited states were performed using two wave function based methods; the approximate coupled-cluster singles-and-doubles method (CC2) (Ref. 53) and CASPT2. ${ }^{23,24}$ The CC2 method is based on a coupled cluster expansion of a single-determinant Hartree-Fock (HF) reference wave function and generally performs best for systems that are well described by this single-configurational reference. The CASPT2 method, in contrast, performs a configuration interaction calculation on a complete active space of selected orbitals and electrons relevant to the problem at hand and is inherently multiconfigurational.

The CC2 calculations were performed using the RICC2 (Ref. 54) module of TURBOMOLE 5.10 and employed the resolution-of-the-identity approximation to compute the electron repulsion integrals. Both def2-TZVP and def2-TZVPP (Ref. 52) basis-sets were used to expand the reference HF wave function, while the suitability of the single-configuration approximation inherent to $\mathrm{CC} 2$ was tested by the D1 measure of Janssen et al. ${ }^{55}$ For the CASPT2 calculations the MOLCAS-7 package in combination with a def2-TZVPP basis-set was employed. Here, first an $\mathrm{N}$-electron complete active space SCF wave function is computed, which includes an important part of the electron correlation in a variational way, and, in a second step, the remainder, mainly dynamical electron correlation, is obtained by a second order perturbational treatment with the complete active space self-consistent field (CASSCF) wave function as zeroth-order wave function. To define the active space, restricted active space SCF calculations have been performed and the orbitals with the largest deviation from two or zero in the natural orbital occupation numbers have been included in the active space. For the $\mathrm{Zn}_{4} \mathrm{~S}_{4}$ cluster an active space containing 10 orbitals and 12 electrons has been considered, corresponding to the highest occupied $\mathrm{T}_{1}$ and $\mathrm{T}_{2}$ orbitals 
and the lowest virtual orbitals of $A_{1}$ and $T_{1}$ symmetry. The active space for the $\mathrm{Zn}_{6} \mathrm{~S}_{6}$ cluster included six orbitals, the five highest occupied orbitals and the lowest unoccupied orbital, and ten electrons. In the subsequent second order perturbational CASPT2 calculations all valence electrons of both the clusters ( $\mathrm{Zn}-3 s, 3 p, 3 d$, and $4 s$ and $\mathrm{S}-3 s, 3 p)$ have been correlated.

\section{RESULTS AND DISCUSSION}

\section{A. Vertical excitation}

Optical absorption spectra for small polyhedral $\mathrm{ZnS}$ clusters have been previously studied by Matxain and coworkers ${ }^{44-46}$ however, they report only very limited detail on these spectra. As a good knowledge of the vertical excitation spectra is critical when exploring the subsequent localization of the electron density in the excited state, we decided to reinvestigate the vertical excitation spectra of $Z_{4} S_{4}$ and $Z_{6} S_{6}$ with a combination of TD-B3LYP, CC2, and CASPT2. This further gives us the opportunity to validate the suitability of TD-B3LYP for predicting the excited state properties of polyhedral $\mathrm{ZnS}$ clusters by comparing TD-B3LYP and wave function based results.

Table I gives the lowest excitations for $\mathrm{Zn}_{4} \mathrm{~S}_{4}$ obtained from TD-B3LYP, TD-PBE, TD-BHLYP, CC2, and CASPT2 calculations and allows for two clear observations. First, the CASPT2 and CC2 calculated excitation energies lie slightly higher than those obtained by TD-B3LYP, (difference less than $0.25 \mathrm{eV}$ for the first two excitations and less than 0.3 $\mathrm{eV}$ for the higher excitations in CASPT2), and follow the same energetic ordering. It should be noted that the basisset used for CASPT2 and most CC2 calculations is slightly larger, def2-TZVPP instead of the def2-TZVP used in the TDDFT calculations (i.e., one $d$-polarization function extra for $\mathrm{S}$ and an extra $f$ - and one $g$-polarization function for $\mathrm{Zn}$ ), as correlated wave function methods are more sensitive to the addition of further polarization functions. This small difference in basis-sets does not lead to any extra ambiguity when comparing TD-DFT and wave function based results as the TD-B3LYP results do not significantly change when increasing the basis-set from def2-TZVP to def2-TZVPP (differ-

TABLE I. The lowest excitations for the $\mathrm{Zn}_{4} \mathrm{~S}_{4}$ polyhedral cluster calculated using TD-B3LYP (values in parentheses calculated with the larger def2-QZVP basis-set), TD-PBE, TD-BHLYP, CASPT2, and CC2 (values in parentheses calculated with the smaller def2-TZVP basis-set), and the def2-TZVP basis-set for TD-DFT, and the def2-TZVPP basis-set for CC2 and CASPT2. Excitations highlighted in bold are dipole allowed and carry finite intensity. Oscillator strengths of allowed transitions is given in italics.

\begin{tabular}{llllll}
\hline \hline & TD-B3LYP & TD-PBE & TD-BHLYP & CASPT2 & CC2 \\
\hline $\mathrm{T}_{1}$ & $2.88(2.88)$ & 2.62 & 3.60 & 3.01 & $3.13(3.18)$ \\
$\mathbf{T}_{2}$ & $\mathbf{2 . 9 8 ( 2 . 9 8 )}$ & $\mathbf{2 . 7 0}$ & $\mathbf{3 . 7 2}$ & $\mathbf{3 . 2 2}$ & $\mathbf{3 . 2 1}(\mathbf{3 . 2 4})$ \\
& $2.42 \times 10^{-2}$ & $5.13 \times 10^{-2}$ & $1.08 \times 10^{-2}$ & $4.41 \times 10^{-2}$ & \\
$\mathrm{~A}_{2}$ & $3.97(3.97)$ & 3.76 & 4.68 & 4.26 & \\
$\mathrm{~T}_{1}$ & $4.02(4.02)$ & 3.79 & 4.68 & 4.31 & \\
$\mathrm{E}$ & $4.06(4.05)$ & & & 4.32 & \\
$\mathrm{~T}_{1}$ & $4.14(4.13)$ & & & \\
\hline \hline
\end{tabular}

TABLE II. The six lowest excitations for the $\mathrm{Zn}_{6} \mathrm{~S}_{6}$ polyhedral cluster calculated using TD-B3LYP (values in parentheses are calculated with the larger def2-QZVP basis-set), CASPT2, and CC2 (values in parentheses are calculated with the smaller def2-TZVP basis-set), and a def2-TZVP basisset for TD-DFT, and a def2-TZVPP basis-set for CC2 and CASPT2. The TD-MPW1PW91/SKBJ(D) results of Matxain and co-workers (Ref. 45) added for comparison. Excitations highlighted in bold are dipole allowed and carry finite intensity. Oscillator strengths of allowed transitions is given in italics.

\begin{tabular}{lllll}
\hline \hline & TD-B3LYP & TD-MPW91 & CASPT2 & CC2 \\
\hline $\mathrm{E}_{\mathrm{g}}$ & $3.08(3.07)$ & 3.37 & 3.29 & $3.40(3.43)$ \\
$\mathbf{A}_{\mathbf{2 u}}$ & $\mathbf{3 . 3 4}(\mathbf{3 . 3 3})$ & $\mathbf{3 . 6 0}$ & $\mathbf{3 . 4 4}$ & $\mathbf{3 . 6 7}(\mathbf{3 . 7 0})$ \\
& $2.92 \times 10^{-2}$ & $2.9 \times 10^{-2}$ & $4.44 \times 10^{-2}$ & \\
$\mathrm{E}_{\mathrm{g}}$ & $3.81(3.80)$ & & 4.06 & \\
$\mathbf{E}_{\mathbf{u}}$ & $\mathbf{3 . 8 5}(\mathbf{3 . 8 5})$ & & & \\
& $9.92 \times 10^{-3}$ & & & \\
$\mathrm{~A}_{2 \mathrm{~g}}$ & $3.95(3.95)$ & & & \\
$\mathbf{E}_{\mathbf{u}}$ & $\mathbf{4 . 0 4 ( 4 . 0 2 )}$ & & & \\
& $6.62 \times 10^{-6}$ & & & \\
$\mathbf{E}_{\mathbf{u}}$ & $\mathbf{4 . 1 4 ( 4 . 1 3 )}$ & & & \\
& $1.26 \times 10^{-1}$ & & & \\
\hline \hline
\end{tabular}

ences smaller than $0.01 \mathrm{eV}$, see also below). Second, the GGA (TD-PBE) results lie $\sim 0.25$ eV below the TD-B3LYP results, while the excitation calculated with the BHLYP XC-potential (50\% Fock exchange), with twice the percentage of Fock exchange in B3LYP $(20 \%)$, lie consistently $\sim 0.7 \mathrm{eV}$ higher in energy. In the case of $\mathrm{Zn}_{6} \mathrm{~S}_{6}$ (see Table II) we observe a similar match between TD-B3LYP, CASPT2, and CC2 as found for $\mathrm{Zn}_{4} \mathrm{~S}_{4}$, but also find that our calculated values for the two lowest excitations compare reasonably well with the previously published results by Matxain and co-workers ${ }^{45}$ (whose values lie consistently $\sim 0.3 \mathrm{eV}$ higher than ours). Matxain and co-workers employed the MPW1PW91 (or MPW91) XC potential, with $25 \%$ Fock exchange comparable to B3LYP, and the SKBJ(D) (Ref. 56) basis-set/effective core-potential. The latter basis is double-zeta and only has $d$ polarization functions and is thus small compared with the triple-zeta allelectron def2-TZVP basis-set we use, which has multiple $d$ and one $f$ polarization function. Finally, Tables I and II show that the absorption onset (i.e., the lowest excitation that carries intensity) of both clusters lies on the border between visible (violet) and ultraviolet light. The obtained absorption onset values are low compared with the majority of those measured experimentally for much larger nanostructures, which lie properly in the ultraviolet (see Sec. I), but are roughly comparable to that of the large wavelength absorption feature observed by Chen et al. ${ }^{32}$

The relative weights of the different configurations used in the CASSCF reference for the CASPT2 calculations strongly suggest that in both clusters the excited states can be described as essentially single electron excitations from the dominant ground state wave function configuration. In other words both systems can be accurately described with a single HF reference wave function, which explains the observed close match between single-configurational CC2 and multiconfigurational CASPT2 results, and is probably also instrumental to the observed success of TD-DFT in describ- 
ing the excited states. We would also like to stress that for all methods, both TD-DFT and wave function based methods, the same excitations (when labeled with their irreducible representation) are found to lie lowest in energy and their energetic ordering to be also the same. We thus find no spurious excited states in the TD-DFT calculations that are erroneously stabilized when compared to correlated wave function results.

In both clusters the found excitations correspond to excitations from $3 p$-orbitals on the $\mathrm{S}$ atoms to $4 s$-orbitals on the $\mathrm{Zn}$ atoms. This corresponds with the fact that the highest occupied molecular orbital (HOMO) in both clusters is located principally on the $\mathrm{S}$ atoms and the lowest unoccupied molecular orbital lies mainly on the $\mathrm{Zn}$ atoms. The latter is perhaps not surprising as in the bulk zinc-blende structure the top of the valence band is also predominantly located on $\mathrm{S}$ atoms and the bottom of the conduction band on primarily $\mathrm{Zn}$ atoms. ${ }^{57,58}$

On a more technical note, Tables I and II show that increasing the quality of the basis-set used in the TD-B3LYP calculations beyond the already large def2-TZVP basis-set has only a very minor effect as the differences between the results obtained by the def2-TZVP basis-set and def2-QZVP basis-set are consistently smaller than $0.02 \mathrm{eV}$. This observation is further supported by the fact that if one instead uses a Dunning aug-cc-pVTZ basis-set, which has more diffuse exponents for the outer orbitals, again excitation energies that only differ by up to $0.02 \mathrm{eV}$ with respect to the def2-TZVP result.

Figure 2 shows the TD-B3LYP/def2-TZVP calculated optical excitation spectra up to $7 \mathrm{eV}$ for $\mathrm{Zn}_{4} \mathrm{~S}_{4}$ and of $\mathrm{Zn}_{6} \mathrm{~S}_{6}$, represented as Gaussians broadened by $0.05 \mathrm{eV}$ to mirror vibrational broadening and a height proportional to the computed oscillator strength. Figure 2 shows that the low energy part of the spectra (up to $\sim 5 \mathrm{eV}$ ) of both clusters is made of well-resolved peaks. The higher energy peaks for $\mathrm{Zn}_{6} \mathrm{~S}_{6}$ start to overlap but for $\mathrm{Zn}_{4} \mathrm{~S}_{4}$ stay well defined. The latter is most likely a direct result of the higher symmetry of the $\mathrm{Zn}_{4} \mathrm{~S}_{4}$ cluster. The low energy part of the spectra and the majority of the higher energy peaks in Fig. 2 lie below $-\varepsilon_{\text {HOMO }}$ (the negative of the highest occupied molecular orbital Kohn-Sham energy: $-7 \mathrm{eV}$ for $\mathrm{Zn}_{4} \mathrm{~S}_{4}$ and $-6.8 \mathrm{eV}$ for $\mathrm{Zn}_{6} \mathrm{~S}_{6}$ in B3LYP)

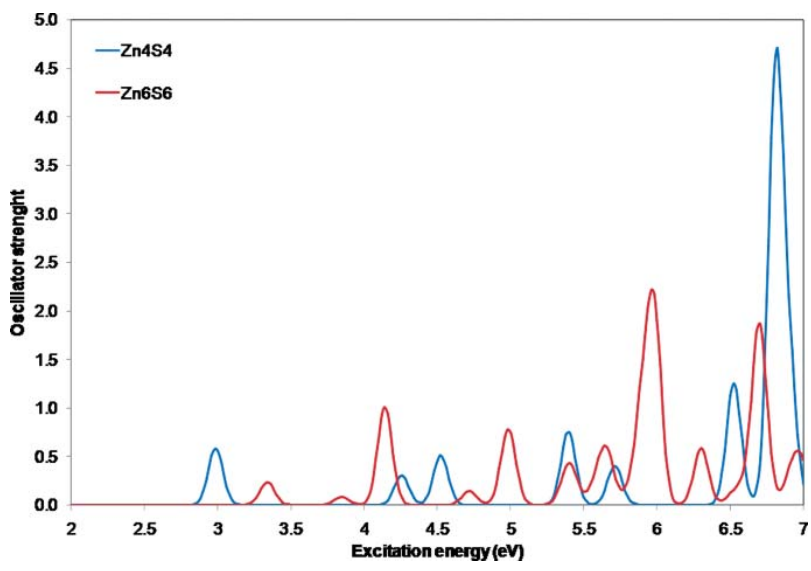

FIG. 2. The optical absorption spectra of $\mathrm{Zn}_{4} \mathrm{~S}_{4}$ and $\mathrm{Zn}_{6} \mathrm{~S}_{6}$ up to $7 \mathrm{eV}$ calculated with TD-B3LYP and a def2-TZVP basis-set. suggesting that errors due to the incorrect long-range behavior of the XC potential ${ }^{59}$ are unlikely to seriously complicate this simple picture. The only possible complication arises from finite temperature effects that, through structural distortions, give dipole forbidden transitions intensities, and lead to extra peaks beyond those in Fig. 2.

\section{B. Localization in the excited state}

To study the localization of the electron density in the excited state, corresponding to an excited electron and the formation of a localized hole, the geometry of the excited state (e.g., the lowest excited singlet S1) has to be energy minimized using TD-DFT. This yields the geometry of the excited state minimum, the total energy of the relaxed excited state [energy of the nth singlet excited state- $\mathrm{Sn}-$ at the minimum energy geometry for $\mathrm{Sn}: \mathrm{E}\left(\mathrm{Sn} / \mathrm{Sn}_{\min }\right)$, where $n>0]$ and the energy of the ground state $(\mathrm{S} 0)$ at the relaxed excited state geometry [Energy of S0 at the minimum energy geometry of $\mathrm{Sn}$ : $\left.\mathrm{E}\left(\mathrm{S} 0 / \mathrm{Sn}_{\min }\right)\right]$. Where the latter two energies and their counterparts for the ground state geometry [i.e., $\mathrm{E}\left(\mathrm{S} 0 / \mathrm{S}_{\min }\right)$ and $\mathrm{E}\left(\mathrm{Sn} / \mathrm{S} 0_{\text {min }}\right)$ ] can be subsequently used to calculate the vertical emission energy at the excited state minimum $\left[\mathrm{E}\left(\mathrm{Sn} / \mathrm{Sn}_{\min }\right)-\mathrm{E}\left(\mathrm{S} 0 / \mathrm{Sn}_{\min }\right)\right]$, the stabilization of the excited state $\left[\mathrm{E}\left(\mathrm{Sn} / \mathrm{S}_{\min }\right)-\mathrm{E}\left(\mathrm{Sn} / \mathrm{Sn}_{\min }\right)\right]$, and the destabilization of the ground state $\left[\mathrm{E}\left(\mathrm{S} 0 / \mathrm{S}_{\min }\right)-\mathrm{E}\left(\mathrm{S} 0 / \mathrm{Sn}_{\min }\right)\right]$.

Both $\mathrm{Zn}_{4} \mathrm{~S}_{4}$ and $\mathrm{Zn}_{6} \mathrm{~S}_{6}$ clusters have degenerate lowlying excited states in their ground state geometry (e.g., the triply degenerate $\mathrm{T}_{1}$ state for $\mathrm{Zn}_{4} \mathrm{~S}_{4}$ ) and are hence likely to suffer from an excited state version of the Jahn-Teller effect. This excited state version of the well-known ground state Jahn-Teller effect has previously been shown to exist, for example, in the similarly cagelike fullerene $\mathrm{C}_{60}$ (using model Hamiltonian approaches ${ }^{60,61}$ ). Because of this excited state Jahn-Teller effect, starting the optimization from this high symmetry geometry would lead to methodological and conceptual problems related to symmetry breaking. Therefore to circumvent such problems we decided to start our excited state geometry optimizations from different starting geometries obtained by distorting the clusters slightly along individual normal modes of vibrations of the ground state geometry (obtained by a frequency calculation on the ground state geometry). These distorted starting geometries generally have lower symmetries and lift the degeneracy between the excited states, even if the resulting splitting between states that otherwise would be degenerate at the ground state geometry is typically still smaller than $0.01 \mathrm{eV}$. Here we exploit the fact that, even if the clusters are highly symmetrical in their athermal ground state, at finite temperatures they distort due to vibrations and lose a large part of their symmetry.

Following the above approach, we optimized the lowest excited singlet state (S1) of $\mathrm{Zn}_{4} \mathrm{~S}_{4}$ and found that, independently of the original applied distortion (i.e., along which normal mode of the ground state), the optimized lowest excited state always lies $0.65 \mathrm{eV}$ lower in energy than at the ground state geometry. Also, the localization, as probed by the difference in Mulliken charges of the ground and excited state, is always the same. In the $\mathrm{S} 1$ excited state, one electron localizes on two of the four $\mathrm{Zn}$ atoms, while a hole 
gets trapped on two of the four $\mathrm{S}$ atoms. The only real difference between the results found with the different starting geometries is on exactly which pairs of atoms the trapping occurs. Following each of the normal modes of the twelve lowest ground state frequencies, we observe localization on each of the combinatorially possible pairs of atoms. Increasing the basis-set to def2-QZVP gives a very similar stabilization of the excited state (by $0.64 \mathrm{eV}$ ) and geometries. The same holds for calculations with the PBE and BHLYP XC potential which while giving substantially different vertical excitation energies (see above) yield very similar stabilizations of the excited state (by 0.65 and $0.66 \mathrm{eV}$, respectively) and localizations. CASPT2 and $\mathrm{CC} 2$ single point calculations on the TD-B3LYP/def2-TZVP optimized geometry finally give slightly smaller stabilization energies ( 0.30 and $0.53 \mathrm{eV}$, respectively) but reiterate the picture that there is a significant energetic driving force for electron density localization.

The biggest changes in the geometry of the $\mathrm{Zn}_{4} \mathrm{~S}_{4}$ cluster upon relaxation of the excited state are, unsurprisingly, around the two $\mathrm{Zn}$ and two $\mathrm{S}$ atoms upon which the electron and hole localize, with the $\mathrm{Zn}-\mathrm{Zn}$ distance increasing by $28 \%$ and the S-S distances decreasing by $16 \%$ (using TD-B3LYP, see, Fig. 3). While the environment of the two $S$ atoms is symmetrical, the environment of the two $\mathrm{Zn}$ atoms is not. One $\mathrm{Zn}$ atom has two $\mathrm{Zn}-\mathrm{S}$ bonds that are $5 \%$ elongated and one that is longer by $1 \%$, while the other $\mathrm{Zn}$ atom has two $\mathrm{Zn}-$ $\mathrm{S}$ bonds that are marginally longer than in the ground state and one that is $2 \%$ shortened. This difference in environment is also mirrored in the Mulliken charges, which suggest that the excited electron is more localized on the former $\mathrm{Zn}$ atom than on the latter. Overall, most of the symmetry of the cluster is lost, with only a mirror plane remaining that goes through the two $\mathrm{S}$ atoms on which the excited state localizes and two $\mathrm{Zn}$ atoms. A symmetric transition state connecting this low

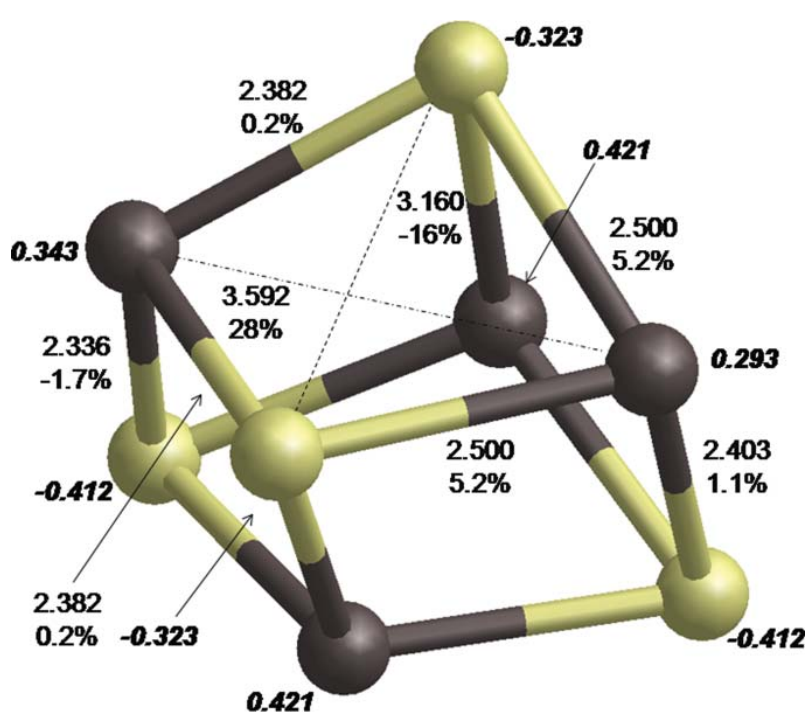

FIG. 3. View of the $\mathrm{Zn}_{4} \mathrm{~S}_{4}$ polyhedral cluster optimized in its lowest singlet excited state by TD-B3LYP/def2-TZVP. Selected distances and Mulliken charges (in bold) displayed, as are the percentage changes in bond length when compared with those in the ground state structure. symmetry structure and a more symmetric one, and in which the two $\mathrm{S}$ atoms are equivalent, lies less than $1 \mathrm{meV}$ above the asymmetric geometry, strongly suggesting that the distortional mode associated with this final symmetry breaking is extremely soft.

TD-PBE and TD-BHLYP minimizations and the CASPT2 single point calculation on the minimum obtained with TD-B3LYP paint essentially the same picture as found with TD-B3LYP. The main difference is the degree of asymmetry in the localization of the electron over the two $\mathrm{Zn}$ atoms. More specifically, this asymmetry expressed as the ratio between their Mulliken excited state charges, increases with the amount of Fock-exchange in the XC-potential from essentially 1 for TD-PBE to 1.17 for TD-B3LYP and 1.29 for TD-BHLYP. We believe the combination of this spread in the Mulliken charge ratio for the different $\mathrm{X}-\mathrm{C}$ potentials with the extremely similar stabilization energies discussed above $(0.65,0.65$, and $0.66 \mathrm{eV})$ reflect again that the distortional mode associated with the symmetry breaking between the two zinc atoms on which the electron localizes is extremely soft.

The case of $\mathrm{Zn}_{6} \mathrm{~S}_{6}$ is more complicated. Using the same procedure as set out for $\mathrm{Zn}_{4} \mathrm{~S}_{4}$ we find three different minima for the lowest excited state singlet [see Figs. 4(a)-4(c)], for which the optimized lowest excited singlet state lies between 0.78 and $1.08 \mathrm{eV}$ lower in energy than at the ground state geometry. The localization, as probed by the difference in Mulliken charges of the ground and excited state, is for all three different minima essentially the same; one electron localizes on a $\mathrm{Zn}$ atom and one hole on a $\mathrm{S}$ atom. The main difference between the three minima is the distance between the $\mathrm{Zn}$ atom on which the hole localizes and on which $\mathrm{S}$ atom the electron. In the case of the most stable minimum (1.08 eV stabilization), one electron and one hole localize on an adjacent pair of $\mathrm{Zn}$ and $\mathrm{S}$ atoms, located along one of the $\mathrm{Zn}-\mathrm{S}$ distances that separates the two $\mathrm{Zn}_{3} \mathrm{~S}_{3}$ rings in the cluster. The latter distance elongates dramatically upon localization [by more than 70\%, see Fig. 4(a)], resulting effectively in a cluster with two two-coordinated atoms. Exploratory calculations using TD-PBE and TD-BHLYP for this minimum yield exactly the same picture, including localization, strongly suggesting that this picture is again independent of the amount of Fock-exchange in the XC-potential used. In the case of the second most stable minimum $[0.84 \mathrm{eV}$ stabilization, see Fig. 4(b)], one electron and one hole localize on a pair of $\mathrm{Zn}$ and $\mathrm{S}$ atoms, located along the diagonal between the two $\mathrm{Zn}_{3} \mathrm{~S}_{3}$ rings in the cluster. In this case the distance between the atoms on which the hole and electron localize is larger $(5.24 \AA$ instead of $4.24 \AA$ ) and we observe that for both these atoms at least bond elongates dramatically (more than 15\%), with the biggest change around the $\mathrm{S}$ atom (where one bond distance elongates by $62 \%$ ). Finally, for the third most stable minimum shown in Fig. 4(c) (0.78 eV stabilization), one electron and one hole localize on a pair of $\mathrm{Zn}$ and $\mathrm{S}$ atoms, located along the diagonal of one $\mathrm{Zn}_{3} \mathrm{~S}_{3}$ ring. Here the distance between the atoms on which the hole and electron localize is $4.72 \AA$ and we observe again that around both the atoms on which the excited states localizes at least of the bonds elongates dramatically (more than 15\%), with the biggest change around the $\mathrm{S}$ 

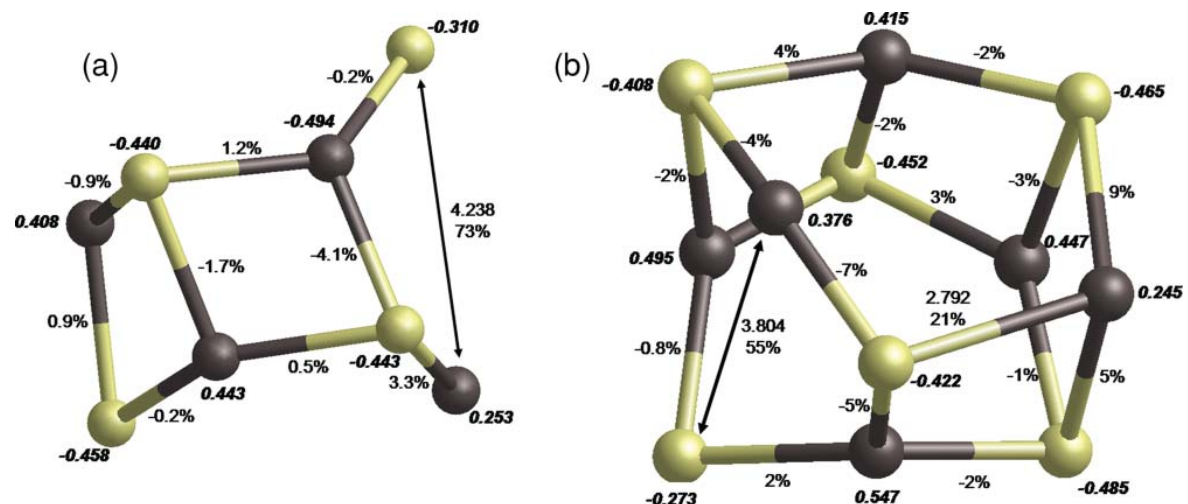

(c)

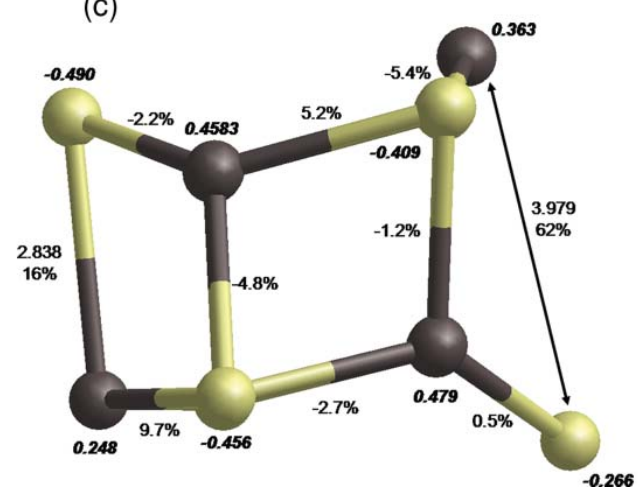

FIG. 4. View of the three minima found for the $\mathrm{Zn}_{6} \mathrm{~S}_{6}$ polyhedral cluster optimized in its lowest singlet excited state by TD-B3LYP/def2-TZVP. Selected distances and Mulliken charges (in bold) displayed, as are the percentage changes in bond length when compared with those in the ground state structure.

atom (where one bond distance elongates by 55\%). A fourth possible minimum, where the electron and hole would localize on a pair of $\mathrm{Zn}$ and $\mathrm{S}$ atoms located along one of the $\mathrm{Zn}-\mathrm{S}$ distances in one of the $Z_{3} S_{3}$ rings, was not found by us (after more than 12 energy minimizations started from uniquely distorted ground state structures) but might exist.

Figure 4(a) shows that when the hole and electron localize on adjacent atoms all further structural distortions larger than $2 \%$ in the excited state minimum are concentrated within two $\mathrm{Zn}-\mathrm{S}$ distances from this pair of atoms and that thus even in this rather small cluster the induced distortions dampen out quickly. Figures 4(b) and 4(c), however, demonstrate that when the hole and electron localize on atoms that are further apart, a large number of the bonds in the cluster are considerably distorted (distortion compared to the ground state geometry of $2 \%-15 \%)$.

\section{Photoluminescence}

Table III gives the spectroscopic signature of the relaxed excited state for $\mathrm{Zn}_{4} \mathrm{~S}_{4}$. The vertical emission energy of the lowest excited state lies in between $0.79 \mathrm{eV}$ (TD-PBE) and $1.53 \mathrm{eV}$ (TD-BHLYP). Just as for the vertical excitation spectra, the vertical emission results of TD-B3LYP and the wave function based methods (CASPT2, CC2) are very similar, although the differences are now slightly larger (the wave function based results lie $\sim 0.2-0.3 \mathrm{eV}$ higher than those ob- tained with TD-B3LYP instead of $\sim 0.1 \mathrm{eV}$ ). Also just as is the case for the ground state geometry, the TD-PBE values lie lower, and the TD-BHLYP values higher in energy than those obtained by TD-B3LYP. The Stokes shift (the difference between the vertical absorption energy at the ground state geometry and the vertical emission at the relaxed excited state geometry) is found to range between $1.71-2.07 \mathrm{eV}$. This value is much larger than the stabilization of the excited state, because of the sizable destabilization of the ground state in the relaxed excited state geometry (between $-1.26 \mathrm{eV}$, TDB3LYP and $-1.43 \mathrm{eV}, \mathrm{CASPT} 2)$. The latter is rather sensitive to the geometry. For example, the ground state energy at the

TABLE III. The three lowest excitations for the $\mathrm{Zn}_{4} \mathrm{~S}_{4}$ polyhedral cluster optimized in its lowest singlet excited state using TD-B3LYP (values in parentheses are calculated with the larger def2-QZVP basis-set), TD-PBE, TD-BHLYP, CASPT2, and CC2 (values in parentheses are calculated with the smaller def2-TZVP basis-set), and a def2-TZVP basis-set for TD-DFT, and a def2-TZVPP basis-set for CC2 and CASPT2. The bold entries give the excitation energy to the lowest singlet excited state and correspond to the predicted PL energy (oscillator strength of lowest singlet excited state given in italics).

\begin{tabular}{lllllc}
\hline \hline & TD-B3LYP & TD-PBE & TD-BHLYP & CASPT2 & CC2 \\
\hline $\mathbf{A}_{\mathbf{1}}$ & $\mathbf{0 . 9 7}(\mathbf{1 . 0 3})$ & $\mathbf{0 . 7 9}$ & $\mathbf{1 . 5 3}$ & $\mathbf{1 . 2 9}$ & $\mathbf{1 . 1 9}(\mathbf{1 . 2 3})$ \\
& $2.91 \times 10^{-3}$ & $1.97 \times 10^{-3}$ & $5.29 \times 10^{-3}$ & $1.02 \times 10^{-2}$ & \\
$\mathrm{~A}_{1}$ & $2.06(2.10)$ & 1.92 & 2.69 & 2.58 & $2.36(2.40)$ \\
$\mathrm{A}_{1}$ & $2.16(2.17)$ & 1.99 & 2.74 & 2.59 & $2.27(2.42)$ \\
\hline \hline
\end{tabular}


TABLE IV. The three lowest excitations for the $\mathrm{Zn}_{6} \mathrm{~S}_{6}$ polyhedral cluster for the three minima found for its lowest singlet excited state using TDB3LYP/def2-TZVP. The bold entries give the excitation energy to the lowest singlet excited state and correspond to the predicted PL energy (oscillator strength of lowest singlet excited state given in italics).

\begin{tabular}{llll}
\hline \hline & \multicolumn{3}{c}{ TD-B3LYP } \\
\cline { 2 - 4 } & \multicolumn{1}{c}{$4 \mathrm{a}$} & \multicolumn{1}{c}{$4 \mathrm{~b}$} & \multicolumn{1}{c}{$4 \mathrm{c}$} \\
\hline $\mathbf{A}_{\mathbf{1}}$ & $\mathbf{0 . 8 6}$ & $\mathbf{0 . 7 5}$ & $\mathbf{0 . 8 7}$ \\
& $5.57 \times 10^{-2}$ & $2.08 \times 10^{-4}$ & $2.05 \times 10^{-3}$ \\
$\mathrm{~A}_{1}$ & 1.87 & 1.82 & 1.86 \\
\hline \hline
\end{tabular}

geometry of the symmetric transition state discussed above is $0.082 \mathrm{eV}$ lower than that at the corresponding fully relaxed excited state geometry (even while, as noted above, the excited state energy changes by less than $0.001 \mathrm{eV}$ between both structures). Table IV gives the spectroscopic signature of the three minima found for the relaxed excited state of $\mathrm{Zn}_{6} \mathrm{~S}_{6}$. The vertical emission energy lies at $0.86 \mathrm{eV}(4 \mathrm{a}), 0.75 \mathrm{eV}$ (4b), and $0.87 \mathrm{eV}(4 \mathrm{c})$, giving Stokes shift ranging between 2.21 and $2.34 \mathrm{eV}$. Just as for $\mathrm{Zn}_{4} \mathrm{~S}_{4}$, this Stokes shift is much larger than the stabilization of the excited state alone, because again the destabilization of the ground state upon relaxation is significant. It is interesting to compare the size of the latter ( $1.14 \mathrm{eV}$ for $4 \mathrm{a}, 1.50 \mathrm{eV}$ for $4 \mathrm{~b}$, and $1.43 \mathrm{eV}$ for $4 \mathrm{c}$ ) with that for $\mathrm{Zn}_{4} \mathrm{~S}_{4}$ and note that even though at first sight the structural distortion is more pronounced in all the $\mathrm{Zn}_{6} \mathrm{~S}_{6}$ cluster geometries, the destabilization of the ground state when the excited state localizes on adjacent atoms (minimum $4 \mathrm{a}$ ) is actually smaller than that found for $\mathrm{Zn}_{4} \mathrm{~S}_{4}$.

In the ground state the lowest singlet excitations of $\mathrm{Zn}_{4} \mathrm{~S}_{4}$ $\left(\mathrm{T}_{1}\right)$ and $\mathrm{Zn}_{6} \mathrm{~S}_{6}\left(\mathrm{E}_{\mathrm{g}}\right)$ are dipole forbidden and thus carry zero oscillator strength. However, even slight geometrical distortions, such as the one applied at the start of the optimization, give rise to finite oscillator strengths for both clusters. Moreover, taking into account the small energy difference between the lowest and second excited states, especially when compared with the energy difference between the ground and first excited state (especially for $\mathrm{Zn}_{4} \mathrm{~S}_{4}$ ), internal conversion after excitation is expected to lead to a significant population of the lowest excited singlet state and PL from the relaxed state is thus likely to be observable in experiment. The PL energy values predicted here for $\mathrm{Zn}_{4} \mathrm{~S}_{4}$ and $\mathrm{Zn}_{6} \mathrm{~S}_{6}$ are again lower than those measured for the larger particles in experiment. Future work should close the size-gap between theory and experiment by extending this work to larger $\mathrm{ZnS}$ nanostructures, defected $\mathrm{ZnS}$ nanostructures and nanostructures with molecules adsorbed on their surface.

\section{CONCLUSIONS}

We have explored the excited state energy landscape of small polyhedral $\mathrm{ZnS}$ clusters $\left(\mathrm{Zn}_{4} \mathrm{~S}_{4}\right.$ and $\left.\mathrm{Zn}_{6} \mathrm{~S}_{6}\right)$ and demonstrated that upon relaxation of the excited state the nanostructures break symmetry and one electron and one hole localize on a small number of $\mathrm{Zn}$ (electron) and S (hole) atoms. We further showed that TD-DFT/B3LYP gives results that are comparable to those obtained with correlated wave function based methods. We further find that the contributions to the Stokes shift of both the energetic stabilization of the excited state and the energetic destabilization of the ground state are large and significant. Finally, we discuss that degeneracy in the excited states makes the clusters to undergo an excited state Jahn-Teller distortion, which raises numerical problems that are circumvented by starting the optimization of excited states from structures distorted along the ground state normal modes.

\section{ACKNOWLEDGMENTS}

We kindly acknowledge Professor C. R. A. Catlow, Dr. A. A. Sokol, Dr. S. M. Woodley, and the participants of the Benasque TDDFT 2008 summer school for stimulating discussion. M.A.Z. thanks the Spanish Ministry for Science and Innovation for a Juan de la Cierva fellowship while F.I. acknowledges support from the Institució Catalana de Recerca i Estudis Avançats through the 2009 ICREA Academia prize for excellence in research. This study has further been supported by the Spanish Ministry for Science and Innovation (Grant FIS2008-02238), by the Generalitat de Catalunya (Grants 2009SGR1041 and XRQTC), by the Royal Society (International Joint Project), and by the COST-D41 action. Computational time on the computers of the Centre de Supercomputació de Catalunya (CESCA) is gratefully acknowledged.

${ }^{1}$ M. Bruchez, M. Moronne, P. Gin, S. Weiss, and A. P. Alivisatos, Science 281, 2013 (1998).

${ }^{2}$ K. H. Lee, J. Nucl. Med. 48, 1408 (2007)

${ }^{3}$ T. J. Deerinck, Toxicol. Pathol. 36, 112 (2008).

${ }^{4}$ K. S. Leschkies, R. Divakar, J. Basu, E. Enache-Pommer, J. E. Boercker, C. B. Carter, U. R. Kortshagen, D. J. Norris, and E. S. Aydil, Nano Lett. 7, 1739 (2007).

${ }^{5}$ L. J. Diguna, Q. Shen, J. Kobayashi, and T. Toyoda, Appl. Phys. Lett. 91, 023116 (2007).

${ }^{6}$ F. Li, D. I. Son, T. W. Kim, E. Ryu, S. W. Kim, S. K. Lee, and Y. H. Cho, Appl. Phys. Lett. 95, 061911 (2009).

${ }^{7}$ S. Dayal, N. Kopidakis, D. C. Olson, D. S. Ginley, and G. Rumbles, Nano Lett. 10, 239 (2010).

${ }^{8}$ Q. Chen and L. M. Peng, Appl. Phys. Lett. 84, 4920 (2004).

${ }^{9}$ T. J. Lin, H. L. Chen, Y. F. Chen, and S. F. Cheng, Appl. Phys. Lett. 93, 223903 (2008).

${ }^{10}$ R. Kunneth, C. Feldmer, and H. Kisch, Angew. Chem., Int. Ed. 31, 1039 (1992).

${ }^{11}$ H. Fujiwara, H. Hosokawa, K. Murakoshi, Y. Wada, and S. Yanagida, Langmuir 14, 5154 (1998)

${ }^{12}$ G. Horner, P. Johne, R. Kunneth, G. Twardzik, H. Roth, T. Clark, and H. Kisch, Chem.-Eur. J. 5, 208 (1999).

${ }^{13}$ X. X. Yu, J. G. Yu, B. Cheng, and B. B. Huang, Chem. Eur. J. 15, 6731 (2009).

${ }^{14}$ D. Chen, F. Huang, G. Ren, D. Li, M. Zheng, Y. Wang, and Z. Lin, Nanoscale 2, 2062 (2010).

${ }^{15}$ F. Furche and R. Ahlrichs, J. Chem. Phys. 117, 7433 (2002).

${ }^{16}$ F. Furche and R. Ahlrichs, J. Chem. Phys. 121, 12722 (2004).

${ }^{17}$ D. Rappoport and F. Furche, Time Dependent Density Functional Theory, Lecture Notes in Physics Vol. 706 (Springer, Berlin, 2006).

${ }^{18}$ D. Sundholm, Phys. Chem. Chem. Phys. 6, 2044 (2004).

${ }^{19}$ X. Wang, R. Q. Zhang, S. T. Lee, T. A. Niehaus, and Th. Frauenheim, Appl. Phys. Lett. 90, 123116 (2007).

${ }^{20}$ S. Meng, J. Ren, and E. Kaxiras, Nano Lett. 8, 3266 (2008).

${ }^{21}$ Y. Wang, R. Zhang, T. Frauenheim, and T. A. Niehaus, J. Phys. Chem. C 113, 12935 (2009).

${ }^{22}$ M. A. Zwijnenburg, A. A. Sokol, C. Sousa, and S. T. Bromley, J. Chem. Phys. 131, 034705 (2009). 
${ }^{23}$ K. Andersson, P. A. Malmqvist, B. O. Roos, A. J. Sadlej, and K. Wolinski, J. Phys. Chem. 94, 5483 (1990).

${ }^{24}$ K. Andersson, P. A. Malmqvist, and B. O. Roos, J. Phys. Chem. 96, 1218 (1992).

${ }^{25}$ R. Rosesetti, R. Hull, J. M. Gibson, and L. E. Brus, J. Chem. Phys. 82, 552 (1985).

${ }^{26}$ N. Cheetnoy, R. Hill, and L. E. Brus, J. Chem. Phys. 85, 2237 (1986).

${ }^{27}$ A. A. Khosravi, M. Kundu, L. Jatwa, S. K. Deshpande, U. A. Bhagwat, M. Sastry, and S. K. Kulkami, Appl. Phys. Lett. 67, 2702 (1995).

${ }^{28}$ M. Tan, W. Cai, and L. Zhang, Appl. Phys. Lett. 71, 3697 (1997).

${ }^{29}$ W. Vogel, P. H. Borse, N. Deskmukh, and S. K. Kulkarni, Langmuir 16, 2032 (2000).

${ }^{30}$ W. S. Chae, J. H. Yoon, H. Yu, D. J. Jang, and Y. R. Kim, J. Phys. Chem. B 108, 11509 (2004).

${ }^{31}$ D. J. Jovanovic, I. L. Validzic, I. A. Jankovic, N. Bibic, and J. M. Nedeljkovic, Mater. Lett. 61, 4396 (2007).

${ }^{32}$ W. Chen, Z. G. Wang, Z. J. Lin, and L. Y. Lin, J. Appl. Phys. 82, 3111 (1997).

${ }^{33}$ N. A. Dhas, A. Zaban, and A. Gedanken, Chem. Mater. 11, 806 (1999).

${ }^{34}$ W. G. Becker and A. J. Bard, J. Phys. Chem. 87, 4888 (1983).

${ }^{35}$ W. Chen, Z. Wang, Z. Lin, and L. Lin, Appl. Phys. Lett. 70, 1465 (1997).

${ }^{36}$ J. M. Matxain, J. E. Fowler, and J. M. Ulgade, Phys. Rev. A 61, 053201 (2000).

${ }^{37}$ E. Spano, S. Hamad, and C. R. A. Catlow, J. Phys. Chem. B 107, 10337 (2003).

${ }^{38}$ E. Spano, S. Hamad, and C. R. A. Catlow, Chem. Commun. 864 (2004).

${ }^{39}$ S. Woodley, A. Sokol, and C. R. A. Catlow, Z. Anorg. Allg. Chem. 630, 2343 (2004).

${ }^{40}$ S. Hamad, C. R. A. Catlow, and E. Spano, J. Phys. Chem. B 109, 2703 (2005).

${ }^{41}$ E. Sanville, A. Burnin, and J. J. BelBruno, J. Phys. Chem. A 110, 2378 (2006).
${ }^{42}$ S. Hamad and C. R. A. Catlow, J. Cryst. Growth 294, 2 (2006).

${ }^{43}$ R. J. Buenker and S. D. Peyerimhoff, Theor. Chim. Acta 35, 33 (1974).

${ }^{44}$ J. M. Matxain, A. Irigoras, J. E. Fowler, and J. M. Ugalde, Phys. Rev. A 63, 013202 (2000).

${ }^{45}$ J. M. Matxain, A. Irigoras, J. E. Fowler, and J. M. Ugalde, Phys. Rev. A 64, 013201 (2001)

${ }^{46}$ J. M. Matxain, L. A. Eriksson, J. M. Mercero, J. M. Ugalde, E. Spano, S. Hamad, and C. R. A. Catlow, Nanotechnology 17, 4100 (2006).

${ }^{47}$ A. D. Becke, J. Chem. Phys. 98, 5648 (1993).

${ }^{48}$ K. Burke, M. Ernzerhof, and J. P. Perdew, Phys. Rev. Lett. 77, 3865 (1996).

${ }^{49}$ A. D. Becke, J. Chem. Phys. 98, 1372 (1993)

${ }^{50}$ D. E. Woon and T. H. Dunning, Jr., J. Chem. Phys. 98, 1358 (1993).

${ }^{51}$ N. B. Balabanov and K. A. Peterson, J. Chem. Phys. 123, 064107 (2005).

${ }^{52}$ F. Weigend and R. Ahlrichs, Phys. Chem. Chem. Phys. 7, 3297 (2005).

${ }^{53}$ O. Christiansen, H. Koch, and P. Jørgensen, Chem. Phys. Lett. 243, 409 (1995).

${ }^{54}$ C. Hättig and A. Köhn, J. Chem. Phys. 117, 6939 (2002)

${ }^{55}$ C. L. Janssen and I. M. B. Nielsen, Chem. Phys. Lett. 290, 423 (1998).

${ }^{56}$ W. J. Stevens, M. Kraus, H. Basch, and P. Jasien, Can. J. Chem. 70, 612 (1992).

${ }^{57}$ R. Edelbro, Å. Sandström, and J. Paul, Appl. Surf. Sci. 206, 300 (2003).

${ }^{58}$ G. U. von Oertzen, R. T. Jones, and A. R. Gerson, Phys. Chem. Miner. 32 255 (2005).

${ }^{59}$ M. E. Casida, C. Jamorski, K. C. Casida, and D. R. Salahub, J. Chem. Phys. 108, 4439 (1998).

${ }^{60}$ S. Suzuku, D. Inomata, N. Sashide, and K. Nakao, Phys. Rev. B 48, 14615 (1993).

${ }^{61}$ M. Kallay, K. Nemeth, and P. R. Surjan, J. Phys. Chem. A 102, 1261 (1998). 